\title{
Positive results of patch tests with fragrance markers: analysis of a 15- year period at a Brazilian dermatology center*
}

\author{
Mariana de Figueiredo Silva Hafner'1, Sandy Daniele Germano Munhoz², Ariel Garcia Jeldes², Rosana \\ Lazzarini $^{1}$
}

DOI: http://dx.doi.org/10.1590/abd1806-4841.20187743

\begin{abstract}
Fragrances may cause allergic contact dermatitis. Data on patients who were patch tested, between 2000 and 2015, with fragrance allergens included in the Brazilian baseline series (balsam of Peru, colophony and fragrance mix I), were collected and analyzed. Of these patients, 258 (13.8\%) were positive for fragrance markers, 9.8\% being positive for fragrance mix I. Among these 258 there was a predominance of women in their 40s, with hand eczema. The frequency of sensitization to fragrances, as well as the epidemiological profile, was supported by the literature. Fragrance mix I was the main marker. It is important to expand the fragrance markers used in the Brazilian baseline series of patch tests.
\end{abstract}

Keywords: Dermatitis, allergic contact; Patch tests; Perfume

Fragrances are raw materials used to confer or intensify odor. They are widely used in flavoring agents, cleaning products, toys, topical medications and, especially, cosmetics.

Contact with these substances can trigger adverse cutaneous reactions. Studies have demonstrated that fragrances are among the main sensitizers, affecting $1 \%$ of the population. Allergic contact dermatitis (ACD) can result from direct application of the allergen on the skin and mucosae (e.g. deodorants), contact with fomites (e.g. scented pillows), contact with products used by other individuals (e.g. cosmetics used by a partner), and aerial (e.g. perfumes) or systemic (e.g. food flavorings) exposure. ${ }^{1}$

The following fragrance markers are included in the Brazilian baseline series used for patch tests: fragrance mix I (FMI), bal- sam of Peru, and colophony. ${ }^{2}$ However, more than 3,000 fragrances have already been described, and it is therefore probable that ACD caused by fragrance sensitizers has been underdiagnosed.

The current study aimed to verify the frequency of sensitization to fragrance allergens in a Brazilian population group, and the epidemiological profile of this group.

We analyzed, retrospectively, the medical records of patients with positive patch tests for fragrance markers. These tests were performed at the outpatient practice of a Brazilian hospital between January 2000 and December 2015, and followed the methodology established by the International Contact Dermatitis Research Group (ICDRG). Patch tests were applied to the patients' backs using Finn Chambers containers (Epitest Ltd., Oy, Finland). The substances

\footnotetext{
Received 12 October 2017.

Accepted 07 March 2018.

* Work conducted at Santa Casa de Misericórdia de São Paulo, São Paulo (SP), Brazil.

Financial support: None.

Conflict of interest: None.

Dermatology Clinic, Santa Casa de São Paulo, São Paulo (SP), Brazil.

Medical student, Faculdade de Ciências Médicas, Santa Casa de São Paulo, São Paulo (SP), Brazil.
}

MAILING AdDRESS:

Mariana de Figueiredo Silva Hafner

E-mail: marifigs@yahoo.com.br

(C2018 by Anais Brasileiros de Dermatologia 
used were those of the Brazilian baseline series manufactured by the FDA Allergenic (Rio de Janeiro, Brazil).

The collected data pertained to the tests with positive results for one or more fragrance markers at the 96-hour reading (balsam of Peru, colophony and FMI).

During the 2000-2015 period, 1870 patch tests were performed, 258 (13.8\%) of which were positive for fragrance markers and were included in the analysis. This percentage was similar to those observed in the literature: ACD caused by fragrances affects $10 \%$ to $12 \%$ of patients with eczema. ${ }^{3}$ Geographic variations may occur due to differences in the number of allergens tested and their concentrations, as well as to regional preferences for the use of certain fragrances. ${ }^{4,5}$

In relation to the 258 individuals, 60 (31\%) were male and $178(69 \%)$ were female. The average age was 47.4 years. In terms of ethnicity, 142 (55\%) were white; 78 (30.2\%), mixed-race; 35 (13.6\%), black; and $3(1.2 \%)$, yellow. The average evolution time of the dermatosis was 48.2 months. Regarding location of the lesions, the site of highest incidence was the back of the hands, with 82 cases (32\%); the face, with 77 cases (30\%); and the neck, with 71 cases (28\%). These data are compatible with those in the literature on ACD caused by fragrances (though this diagnosis had not been confirmed for all cases): a predominance of women (probably due to greater use of cosmetics) in their 40s, with lesions on the hands, face and neck. ${ }^{3}$

Of the tests that were positive for fragrance markers, 183 (70.9\%) were positive for FMI; 54 (20.1\%), for balsam of Peru; and 83 $(32.3 \%)$, for colophony. Some patients had more than one positive test: $30(11.6 \%)$ for balsam of Peru and FMI; 18 (7\%) for balsam of Peru and colophony; 26 (10\%) for FMI and colophony; and 12 (4.7\%) for all three markers.

The positive tests for FMI represented 9.8\% (183/1870) of the total, a finding consistent with those of European studies (4\% to $11 \%){ }^{5-7}$ The positive results for balsam of Peru constituted $2.8 \%$ $(54 / 1870)$ of the tests, also compatible with the results in other pub- lications (1.6\% to $10.8 \%)$. ${ }^{1,3,7,8}$ The two markers together represented $207(80.3 \%)$ of the tests for fragrance markers. Although the studies show that tests for FMI and balsam of Peru are effective in detecting $70 \%$ to $90 \%$ of the cases of ACD cause by fragrances, new markers have also gained inportance in the European series. ${ }^{4}$

After the relevance of the positive tests was established, 189 patients presented a final diagnosis of ACD. The main etiologies were cosmetics, with 71 cases (37.5\%), and topical medications, with 44 cases $(23.3 \%)$. However, in 53 (28\%) of the tests, the relevance of positivity for fragrance markers could not be determined.

The studies show that fragrances are the main cause of allergy to cosmetics. ${ }^{4}$ They can also act in topical medications as sensitizers: one publication showed that $3.8 \%$ of 3.378 cases of SCD caused by medications occurred due to fragrances. ${ }^{9}$

In 2003, the legislation of the European Union was adjusted such that 26 fragrances now require labeling on products. ${ }^{3}$ In Brazil, the mandatory requirements for labeling of cosmetics are found in the RDC n. ${ }^{\circ} 211 / 2005$ and include the necessary detailing of a product's entire composition. However, a Brazilian study demonstrated that, of the products analyzed by the Fundação Oswaldo Cruz National Institute of Quality Control in Health, 94\% presented labeling in contravention of the Brazilian legislation in effect. ${ }^{10}$

The lack of knowledge regarding the exact composition of products makes establishing the relevance of patch tests difficult. Thus, the recommendation is to complement the investigation with patient's own products (provided they are not rinsable). The use test (ROAT) is performed by applying the product on a $5-\mathrm{cm} \times 5-\mathrm{cm}$ patch on the forearms, twice daily for one week. The presence of an inflammatory reaction suggests that the product is the cause of $\mathrm{ACD}$, though this does not specify the allergen.

Thus, fragrances are important sensitizers in our environment. To improve diagnostic possibility, effective compliance with the Brazilian regulations on product labeling is essential, as are further studies to broaden the range of fragrance markers used for patch tests in Brazil. $\square$ 


\section{REFERENCES}

1. Cheng J, Zug KA. Fragrance allergic contact dermatitis. Dermatitis. 2014;25:232-45.

2. Contact Dermatitis Brazilian Studying Group, "Departamento Especializado de Alergia em Dermatologia da Sociedade Brasileira de Dermatologia. Multicentric study for the development of a standard Brazilian patch test series. An Bras Dermatol. 2000;75:147-56.

3. Johansen JD. Fragrance contact allergy: a clinical review. Am J Clin Dermatol. 2003:4:789-98

4. Arribas MP, Soro P, Silvestre JF. Allergic contact dermatitis to fragrances. Part 1. Actas Dermosifiliogr. 2012;103:874-9.

5. Uter W, Geier J, Frosch P, Schnuch A. Contact allergy to fragrances: current patch test results (2005-2008) from the Information Network of Departments of Dermatology. Contact Dermatitis. 2010;63:254-61.

6. Nardelli A, Carbonez A, Drieghe J, Goossens A. Results of patch testing with fragrance mix 1, fragrance mix 2, and their ingredients, and Myroxylon pereirae and colophonium, over a 21-year period. Contact Dermatitis. 2013;68:307-13.
7. Frosch PJ, Duus Johansen J, Schuttelaar ML, Silvestre JF, Sánchez-Pérez J Weisshaar E, et al. Patch test results with fragrance markers of the baseline series - analysis of the EuropeanSurveillance System on Contact Allergies (ESSCA) network 2009-2012. Contact Dermatitis. 2015;73:163-71.

8. Thyssen JP, Menné T, Linneberg A, Johansen JD. Contact sensitization to fragrances in the general population: a Koch's approach may reveal the burden of disease. Br J Dermatol. 2009;160:729-35.

9. Nardelli A, D'Hooghe E, Drieghe J, Dooms M, Goossens A. Allergic contact dermatitis from fragrances components in specific topical pharmaceutical products in Belgium. Contact Dermatitis. 2009;60:303-13.

10. Rito PN, Presgrave RF, Alves EN, Huf G, Villas Bôas MHS. Avaliação dos aspectos do controle da qualidade de produtos cosméticos comercializados no Brasil analisados pelo Instituto Nacional de Controle de Qualidade em Saúde. Rev Inst Adolfo Lutz. 2012;71:557-65

\section{AUTHORS'CONTRIBUTIONS}

Mariana de Figueiredo Silva Hafner

(iD) ORCID 0000-0001-8322-3856

Approval of the final version of the manuscript, Elaboration and writing of the manuscript, Effective participation in research orientation, Critical review of the literature

Sandy Daniele Germano Munhoz

(iD) ORCID 0000-0003-4244-1528

Obtaining, analyzing and interpreting the data

Ariel Garcia Jeldes

Obtaining, analyzing and interpreting the data

Rosana Lazzarini

(iD ORCID 0000-0002-9032-9533

Conception and planning of the study, Elaboration and writing of the manuscript, Effective participation in research orientation, Critical review of the literature

How to cite this article: Hafner MFS, Munhoz SDG, Jeldes AG, Lazzarini R. Positive results of patch tests with fragrance markers: analysis of a 15-year period at a Brazilian dermatology center. An Bras Dermatol. 2018;93(6):910-2. 